\title{
Hemodynamic Response Function in Absence Epilepsy: An EEG-fMRI Study
}

\author{
Kaiqing Xue ${ }^{1, a,{ }^{*}, \text { Xue Tang }}{ }^{2, b}$ \\ ${ }^{1}$ School of Computer Science and Software Engineering, Xihua University, Chengdu, China \\ 2 Jinjiang Maternal and Child Health Hospital, Chengdu, China \\ a, ${ }^{*}$ xuekaiqing@163.com \\ b394631289@qq.cn
}

Keywords: Hemodynamic Response Function, Absence Epilepsy, EEG-fMRI

Abstract. Understanding hemodynamic response function (HRF) is a key issue for exploring the underlying dynamics of nervous system activation. In this study, epilepsy discharges were adopted as markers of short stimuli, and then EEG-fMRI method was used to investigate HRF in absence epilepsy. Five patients with absence epilepsy were enrolled and their HRF was estimated. The results show that the delay of the estimated HRF is different from either one of the three theoretical model, thus further study on both theoretical model and real data estimation is needed.

\section{INTRODUCTION}

Hemodynamic response function (HRF) describes the dynamic process of the blood oxygen level dependent (BOLD) signal evoked by a very short stimulus of unit intensity [1]. The shape of the HRF varied with different brain regions and different subjects, even at different time in the same brain region of a subject [2]. Past majority studies assumes a fixed HRF modeled by Gamma-variate function, Glover function or the SPM's canonical HRF function [3].

(1) Gamma HRF ( Eq. 1):

$$
h_{\text {gam }}(t)=\left(\frac{t}{b c}\right)^{b} \times e^{b-\frac{t}{c}}
$$

where $b=8.6, c=0.547$. The peak delay is $4.7 \mathrm{~s}$.

(2) Glover HRF ( Eq. 2):

$$
h_{g l o}(t)=\left(\frac{t}{d_{1}}\right)^{a_{1}} \times e^{-\left(\frac{t-d_{1}}{b_{1}}\right)}-c \times\left(\frac{t}{d_{2}}\right)^{a_{2}} \times e^{-\left(\frac{t-d_{2}}{b_{2}}\right)}
$$

where $\mathrm{a}_{1}=6, \mathrm{a}_{2}=12, \mathrm{~b}_{1}=\mathrm{b}_{2}=0.9, \mathrm{c}=0.35$. The first peak(positive) delay is $5.4 \mathrm{~s}$ and the second peak(negative) delay is $10.8 \mathrm{~s}$.

(3) SPM canonical HRF ( Eq. 3):

$$
h_{\text {spm }}(t)=\frac{l_{1} h_{1} \times t^{\left(h_{1}-1\right)} \times e^{-l_{1} t}}{\operatorname{gamma}\left(h_{1}\right)}-c \times \frac{l_{2} h_{2} \times t^{(h 2-1)} \times e^{-l_{2} t}}{\operatorname{gamma}\left(h_{2}\right)}
$$

where $l_{1}=l_{2}=1, h_{1}=6, h_{2}=16, c=2.63$. The first peak(positive) delay is $5.0 \mathrm{~s}$ and the second peak(negative) delay is $9.2 \mathrm{~s}$.

Recently, the simultaneous EEG and functional MRI technique opens the opportunity to assess ongoing brain function related to interictal epileptic discharge (IED). The regions showing fMRI changes presumably involve the epileptogenic zone and the parts that affected by IED[4].Tyvaert and Gotman et al adopted Fourier basis set to discuss the thalamus and its cortical interactions in the 
production and propagation of spike-and-wave discharges. In this work, we investigated HRF in absence epilepsy.

\section{MATERIALS AND METHODS}

\section{Subjects}

Five patients with absence epilepsy (all right-handed) from epilepsy clinics participated for the EEG-fMRI study, then their clinical information was showed in Table 1.

Table 1. Main clinical information for 5 patients

\begin{tabular}{ccccccc}
\hline No. & Gender & Age(year) & $\begin{array}{c}\text { Age of } \\
\text { Onset (year) }\end{array}$ & $\begin{array}{c}\text { Frequency of } \\
\text { Seizure }\end{array}$ & $\begin{array}{c}\text { Antiepileptic } \\
\text { Drugs }\end{array}$ & $\begin{array}{c}\text { Frequency of } \\
\text { SWDs(Hz) }\end{array}$ \\
\hline $\mathbf{1}$ & M & 10 & 5 & $2-3 / \mathrm{d}$ & None & $2.5-3.5$ \\
$\mathbf{2}$ & M & 7 & 4 & $15 / \mathrm{d} ;$ & VPA & $3-3.5$ \\
$\mathbf{3}$ & M & 11 & 9 & $7-8 / \mathrm{d}$ & None & 3 \\
$\mathbf{4}$ & M & 5 & 2 & several/d & None & 3 \\
$\mathbf{5}$ & F & 9 & 4 & $20 / \mathrm{d}$ & None & 3 \\
\hline
\end{tabular}

Abbreviation: M, male; F, female; d, day; VPA, valproic acid

\section{fMRI Acquisition}

fMRI data were collected using a 3.0 T Siemens scanner (Germany) using snapshot gradient echo-planar imaging (EPI), with parameters as follows: 30 slices, TR $=2000 \mathrm{~ms}, \mathrm{TE}=30 \mathrm{~ms}$, flip angle $=90^{\circ}, \mathrm{FOV}=24 \mathrm{~cm} \times 24 \mathrm{~cm}$, matrix $=64 \times 64$, voxel size $=3.75 \times 3.75 \times 5 \mathrm{~mm}^{3}$ ). For each patient, fMRI scanning lasted $7 \mathrm{~min}$, with 205 volumes recorded in total. During fMRI data acquisition, subjects were required to relax with eyes closed, without falling asleep. According to patient endurance, 2- 5 runs were performed for each subject.

\section{EEG acquisition}

Simultaneously collecting fMRI data, EEG data were continuously recorded in the MRI scanner bore through $18 \mathrm{MRI}$-compatible $\mathrm{Ag} / \mathrm{AgCl}$ electrodes placed on the scalp according to the 10-20 system for each patient. The amplifier was connected to a Mizar 40 (EBNerro, Florence, Italy) encased inside a non-ferrous headbox in the scanner room. The headbox was connected to the recording monitor outside the room through a fiber optic cable. The sampling rate was set at $4 \mathrm{kHz}$ and EEG dynamic range was kept at $\pm 65.5 \mathrm{mV}$ to prevent MRI artifact waveforms.

\section{EEG processing}

The MR artifacts could be filtered offline with the BE-MRI Tool box software (Galileo New Technology, Florence, Italy)[5]. A neurology expert then visually reviewed the artifact-corrected EEG recording and marked all epilepsy discharges as markers of epileptic events for each patient.

\section{fMRI Data preprocessing}

All the preprocessing of fMRI Data was conducted with SPM8, (http://www.fil.ion. ucl.ac.uk/spm). The slice time correction, 3D motion detection and correction, spatial normalization to MNI EPI template and spatial smoothing using an isotropic Gaussian kernel were executed on the remaining 200 volumes[6]. The subject with maximum displacement $>1 \mathrm{~mm}$ at each axis or the angular motion $>1{ }^{\circ}$ were excluded in the following analysis. Then, we used a linear regression process for further reducing the effects of head motion, cerebrospinal fluid (CSF), white matter (WM) and global signal (mean of whole brain signal) [7]. Finally, temporal band-pass filtering $(0.01 \sim 0.08 \mathrm{~Hz})$ was performed on each voxel to reduce the effects of low-frequency drift and high-frequency noise. 


\section{Data analysis}

The EEG-fMRI data in epilepsy usually were analyzed by the general linear model(GLM) framework, which models the prior knowledge of hemodynamic response in the design matrix, and then explained the measured data by parameter estimation. The design matrix embodies usually all available knowledge about experimentally controlled factors and potential confounds. In this study, we used SPM8 software package and Matlab software, and the design matrix was made up of the following two sets of regressors: (1)Fourier set: Regressors were procured by convolving a time series of IED time markers with 8 cosines and sines, which are modulated by a Hanning window, over $32 \mathrm{~s}$ time-windows starting at the event onsets. This model assumes that the hemodynamic response can be modeled as the output from a linear low-pass system when an impulse is used as input.(2) Confounds set: Effects of motion are modeled by including 6 head motion parameters.
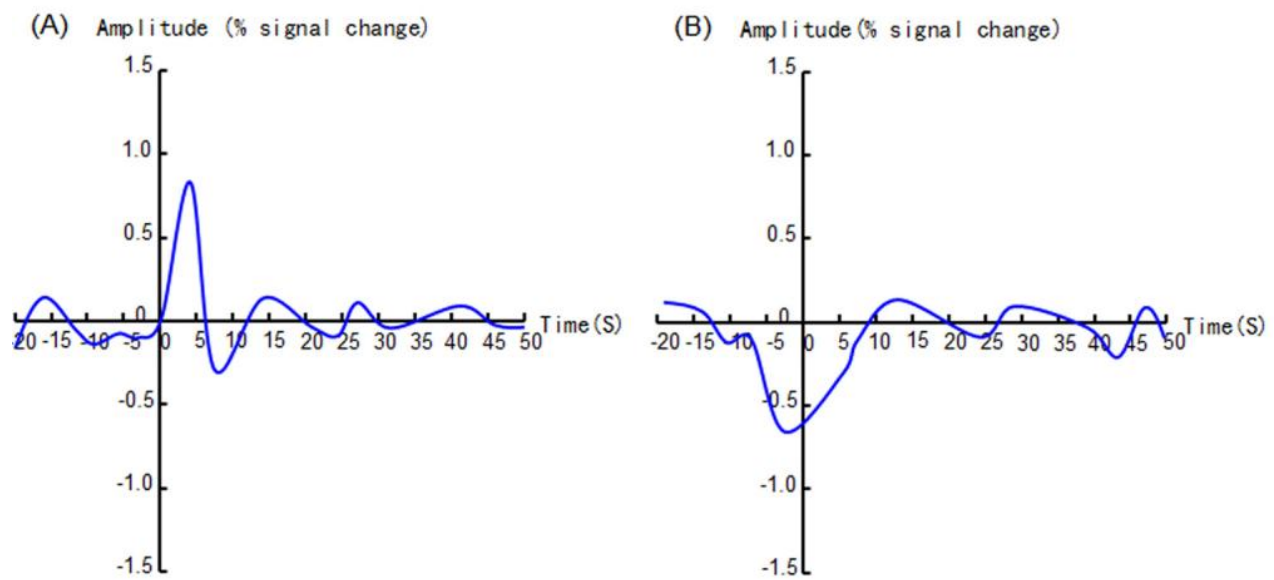

Fig. 1 (A) concordant with canonical HRF;

(B) not concordant with canonical HRF.
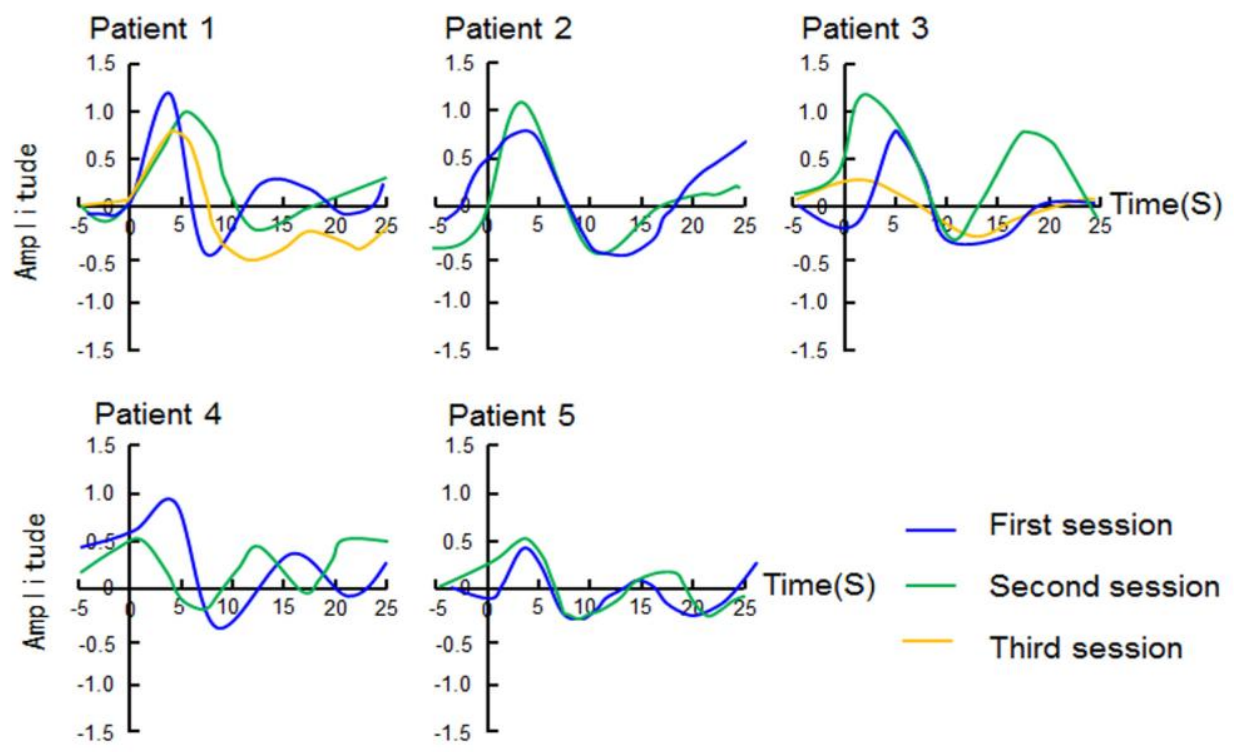

Fig. 2 The time courses are concordant with canonical HRF in all five patients 


\section{Classification and comparison}

The hemodynamic response curves were classififed to two classes: curve concordant with (Fig.1A) or not with (Fig.1B) the standard HRF shape. For comparion with the Gamma HRF, Glover HRF and SPM canonical HRF, the HRF similar to Fig.1A was selected and the one similar to Fig.1B was excluded. Then each selected HRF was characterized by peak delay and amplitude. Then the average and standard deviation of delay and amplitude were calculated for all selected HRF, as shown Fig 2.

\section{RESULTS AND DISCUSSIONS}

Seventeen EEG-fMRI data were collected for 5 patients and were calculated 17 curves, and 12/17 are concordant with the standard HRF shape. The results of remained HRF are as the follow : positive peak delay $=4.3 \pm 1.5(\mathrm{~s})$; positive peak amplitude $=0.85 \pm 0.22$; negative peak delay $=8.2 \pm 3.2(\mathrm{~s})$; negative peak amplitude $=-0.45 \pm 0.16$. The delays of the estimated HRFs and the Gamma HRF, Glover HRF and SPM canonical HRF.were showed in Table 2.

Table 2. Statistics of hemodynamic response

\begin{tabular}{ccccc}
\hline Case & Gamma & Glover & SPM & Our work \\
\hline Positive Peak Delay & 4.7 & 5.4 & 5.0 & 4.3 \\
Negative Peak Delay & - & 10.8 & 9.2 & 8.2 \\
\hline
\end{tabular}

\section{CONCLUSIONS}

Based on the estimation of HRF with comparison to the three theorectical model, it is clear that they all are different to each other, this fact means that the HRF is different for each subjects, and a good imaging may need an individual HRF, however, how to estimate a reliable individual HRF is still an open problem for the future. In this study, only 5 subjects were recruited due to the strict inclusive criteria and cooperation of these young patients. When more subjects participate in our study, the result will be more valuable and receivability.

\section{ACKNOWLEDGMENTS}

This project was funded by Nature Science Foundation of Xihua University (z1422614), Science Foundation of Education Department of Sichuan (15226431) and Provincial Discipline Open Platform Project (szjj2015-063).

\section{REFERENCES}

[1] G Aguirre, E Zarahn,M D'esposito. NeuroImage, Vol. 8 (1998): 360-369.

[2] DA Handwerker, JM Ollinger,M D'Esposito. NeuroImage, Vol. 21 (2004): 1639-1651.

[3] Y Lu, AP Bagshaw, C Grova, et al. NeuroImage, Vol. 32 (2006): 238-247.

[4] C Luo, Q Li, Y Lai, et al. Human Brain Mapping, Vol. 32 (2011): 438-449.

[5] G Garreffa, M Carn1, G Gualniera, et al. Magnetic resonance imaging, Vol. 21 (2003): 1175-1189.

[6] L Lemieux, H Laufs, D Carmichael, et al. Human Brain Mapping, Vol. 29 (2007): 329-345.

[7] MD Fox, AZ Snyder, JL Vincent, et al. Proceedings of the National Academy of Sciences of the United States of America, Vol. 102 (2005): 9673-9678. 